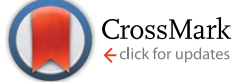

Cite this: RSC Adv., 2015, 5, 23464

Received 31st October 2014 Accepted 23rd February 2015

DOI: 10.1039/c4ra13560a

www.rsc.org/advances

\section{A surface second harmonic generation investigation of volatile organic compound adsorption on a liquid mercury surface $\uparrow$}

\begin{abstract}
Mahamud Subir, ${ }^{* a}$ Nermin Eltouny ${ }^{\mathrm{b}}$ and Parisa A. Ariya*bc
Understanding the adsorption process of volatile organic compounds (VOCs) on various surfaces is essential in the realms of atmospheric, environmental and pollution remediation science. In this study, we investigated the adsorption of selected VOCs (benzene and toluene) on an ideally homogeneous liquid mercury $\left(\mathrm{Hg}_{(1)}\right)$ surface using a surface sensitive nonlinear spectroscopic method of second harmonic generation (SHG). Both of the species investigated showed evidence of reversible physisorption. Determination of SHG adsorption isotherms revealed that attractive adsorbate-adsorbate lateral interaction plays a role in the adsorption of aromatic compounds from the gas phase. Benzene and toluene adsorption has been described by the Hill-de-Boer $(\mathrm{HdB})$ adsorption isotherm model with the corresponding $\mathrm{HdB}$ interaction parameters, $2 \alpha / \beta$, of $2.6 \pm 0.2$ and $3.3 \pm 0.2 \mathrm{kcal} \mathrm{mol}^{-1}$, respectively. Our results highlight the importance of lateral interactions between adsorbed aromatic species at the gas/liquid interfaces. The investigation extends the applicability of SHG to probe complex adsorption processes under ambient conditions.
\end{abstract}

\section{Introduction}

Understanding the adsorption mechanism of atmospheric vapors and gaseous pollutants, such as volatile organic compounds (VOCs), on environmental surfaces including air/ water, air/ice, and aerosol/gas interfaces, is of fundamental and practical importance in the realms of atmospheric, ${ }^{\mathbf{1 , 2}}$ environmental, ${ }^{3,4}$ and pollution remediation science and technology..$^{4-6}$ Knowledge of surface chemistry pertaining to VOCs and gaseous hydrocarbons, such as poly-aromatic hydrocarbons (PAHs) and total petroleum hydrocarbons (TPHs) is essential to elucidate their global transport and fate $\mathrm{e}^{3,7-11}$ in the environment. Further significance of investigating fundamental properties of VOCs adsorption stems out from the need to design efficient adsorbents to remove the airborne pollutants. For example, removal of VOCs using various adsorbent materials has been the subject of numerous chemical engineering studies. ${ }^{4-6}$ In addition, due to the unique adsorptive properties of metal-organic frameworks and its potential application in $\mathrm{CO}_{2}$ capture, a renewed interest in understanding VOC adsorption to these sorbents has also emerged $^{\mathbf{1 2 - 1 5}}$ in recent years.

\footnotetext{
${ }^{a}$ Department of Chemistry, Ball State University, Muncie, IN, 47306, USA. E-mail: msubir@bsu.edu

${ }^{b}$ Department of Chemistry, McGill University, Montreal, QC, H3A 2K6, Canada

${ }^{c}$ Department of Atmospheric and Oceanic Sciences, McGill University, Montreal, QC, H3A 2K6, Canada

$\dagger$ Electronic supplementary information (ESI) available: SHG adsorption and desorption kinetics of three independent runs, isotherm model fit functions, and three different trials of adsorption isotherms. See DOI: 10.1039/c4ra13560a
}

Despite the vast scientific interest to study surface adsorption of VOCs from gas phase to liquid or solid substrates, direct monitoring of the surface species and elucidating the adsorption mechanism at these interfaces has traditionally been challenging. In general, conventional linear spectroscopy, e.g., UV-vis, fluorescence, and IR, has limited application because these techniques do not provide surface selectivity. ${ }^{16,17}$ However, with the advent of nonlinear spectroscopy, and in particular, second harmonic generation (SHG) and vibrational sum frequency generation, probing chemical interfaces under ambient condition has become achievable in recent decades. ${ }^{\mathbf{1 6 - 2 6}}$ Second harmonic generation is a special case of $2^{\text {nd }}$ order nonlinear spectroscopy that exhibits surface specificity and provides information at the molecular level. ${ }^{17,20,23,25,27}$ While the utility of SHG in probing various planar and colloidal surfaces are evident, ${ }^{\mathbf{1 6 , 2 8 , 2 9}}$ advancing its applicability to the study of unique surface interactions remains important. Accordingly, in addition to elucidating the adsorption mechanism and kinetics of atmospherically relevant vapors onto a liquid surface, the underlying aim of the work presented here has been to extend the applicability of SHG to study complex interfacial processes under ambient condition. We have applied this well-established ${ }^{17,25,27}$ surface selective spectroscopic tool to investigate the adsorption of VOCs, namely, benzene and toluene onto ideally homogeneous liquid mercury surface.

The purpose of selecting vapor/mercury interface as a platform to study VOCs interaction is manifold. Elemental mercury is the only metal that is liquid at room temperature and has an "ideal" high energy surface. Based on experimental values ${ }^{30}$ using different measurement techniques, the average surface 
tension value of mercury is $466 \pm 33 \mathrm{dyn}^{-1}$. Liquid mercury, $\mathrm{Hg}_{(1)}$, has an atomically smooth subphase and exhibits shortrange liquid order and atomic mobility. These features are similar to those of water ${ }^{30-32}$ and thus, the chemical interaction at the vapor/mercury interface can serve as a model for the chemistry that takes place at the air/water interface. Moreover, mercury exhibits uniform and homogeneous surface structure and therefore resembles a model homotattic surface, which is an uniform "patch" or region of a larger surface that may or may not be homogeneous as a whole. ${ }^{33}$ These physical attributes of mercury, which are intermediate of air/liquid and air/solid interfaces, render it an ideal surface for adsorption studies.

Due to its significance, there has been a considerable amount of work involving adsorption of organic molecules on the liquid mercury surface based on the macroscopic surface tension measurements. ${ }^{30,34-36}$ Measuring surface tension to elucidate adsorption is a common approach to study adsorption at gas/liquid interface. However, this method is not spectroscopic and does not provide a direct molecular picture of the adsorbate, such as its surface mobility and orientational changes upon binding. Furthermore, it lacks the time resolution to probe real-time surface kinetics. In contrast, SHG is a non-invasive spectroscopic method that is not only sensitive to surface adsorption but also to the orientation of adsorbates. ${ }^{16-26}$ In addition, it provides a sub-millisecond time resolution to monitor adsorption and desorption kinetics, in situ. These advantages provide an additional impetus to probe vapor adsorption on mercury surface using the SHG spectroscopy. Although there are numerous SHG based investigations involving adsorption onto metal surfaces, ${ }^{20-22,37,38}$ to our knowledge there is no SHG investigation of vapor adsorption to mercury surface.

With this multifaceted motivation, we present our exploration of adsorption of benzene and toluene on liquid mercury surface using SHG. We first present an overview of the theoretical aspects of optical SHG from metal surfaces and describe the experimental setup to study in situ vapor adsorption. Thereafter, we provide direct experimental evidence of SHG from the liquid metal surface and its modification due to the adsorption of VOCs from the vapor phase. We also report adsorption and desorption kinetics, which indicate weak physisorption for both benzene and toluene. Based on SHG polarization study, it is determined that the surface structure of liquid mercury remains unaffected upon adsorption. Furthermore, we report SHG adsorption isotherms, which reveal that both of these compounds exhibit a non-Langmuirian adsorption mechanism. This adsorption behavior is markedly different from those of small gas molecule adsorption on clean solid metal surfaces as has been previously observed using SHG. ${ }^{26,39}$ These findings not only advance the applicability of SHG as a surface tool but also provide significant insights into the adsorption process of VOCs onto an ideally homogeneous surface.

\section{Theoretical background}

The process of second harmonic generation involves the interaction of two incident laser fields, each oscillating at frequency $\omega$, with the chemical species to generate a new field at twice the frequency; ${ }^{19,39}$ i.e., at $2 \omega$. In general, a SHG signal exhibits surface specificity and does not include interference from molecules present in the bulk phase because SHG is dipole forbidden in an isotropic medium. ${ }^{17,21,25,27}$ The surface sensitivity arises due to the inherent anisotropic structure of the interfaces. In an adsorption experiment, the total second harmonic response from the metal/gas (substrate-adsorbate) interface can be expressed as: ${ }^{19,21,38}$

$$
E_{\mathrm{SHG}} \propto \chi_{\mathrm{sub}}^{(2)} \mathrm{e}^{\mathrm{i} \varphi}+\chi_{\mathrm{ads}}^{(2)}+\chi_{\mathrm{in} \theta}^{(2)} \theta
$$

In this equation, $E_{\mathrm{SHG}}$, which is the square root of the intensity detected at the SHG wavelength, is the second harmonic field, $\chi_{\text {sub }}^{(2)}, \chi_{\text {ads }}^{(2)}$, and $\chi_{\text {int }}^{(2)}$ are the $2^{\text {nd }}$ order susceptibilities of the substrate, adsorbate, and the substrate-adsorbate interaction, respectively, and $\varphi$ is the relative phase difference between the second order susceptibilities. In our case, the substrate is the neat liquid mercury and the adsorbate corresponds to the vapor introduced into the gas chamber. The underlying assumptions $\mathrm{s}^{21,38}$ in eqn (1) are that the SHG signal scales linearly with surface coverage, $\theta$, and the relative phase difference between the susceptibilities is considered to be independent of $\theta$.

The second order susceptibility is related to the molecular electronic transition..$^{19,39}$ As a result, the SHG signal can be enhanced when the transition between electronic states is in resonance with either the second harmonic or the fundamental frequency of the laser beam. The aromatic compounds investigated in this study exhibit electronic transitions below $300 \mathrm{~nm}$, which is far from the SHG $(401 \mathrm{~nm})$ and fundamental $(802 \mathrm{~nm})$ wavelengths. Thus, given that liquid mercury yields considerable SHG signal (approximately, $10^{2}$ times greater than SHG intensity obtained from air/water interface using the same setup); the contribution from $\chi_{\mathrm{ads}}^{(2)}$ term is negligible. Subsequently, eqn (1) reduces to

$$
E_{\mathrm{SHG}} \propto \chi_{\mathrm{sub}}^{(2)} \mathrm{e}^{\mathrm{i} \varphi}+\chi_{\mathrm{int}}^{(2)} \theta .
$$

The total SHG field (from substrate and adsorbate-substrate interaction) can be further normalized based on the reference SHG signal from the substrate; i.e., $E_{\mathrm{sub}} \propto \chi_{\text {sub }}^{(2)}$ and thus, eqn (2) can be written as

$$
E_{\mathrm{NormSHG}}=1+B \theta
$$

The parameter $B$ is the ratio of the susceptibilities; i.e., $B=\frac{\chi_{\text {int }}^{(2)}}{\chi_{\text {sub }}^{(2)} \mathrm{e}^{\mathrm{i} \varphi}}$. Eqn (3), in conjunction with an existing adsorption isotherm model to describe the $\theta$ dependence on relative pressure, can be used to fit the experimental $E_{\mathrm{NormsHg}} v s$. relative pressure data. This approach of describing SHG adsorption isotherm has been applied previously to study molecular adsorption not only at metal surfaces ${ }^{21,22}$ but also at numerous planar and colloidal interfaces. ${ }^{16-18,24}$ 


\section{Experimental}

Fig. 1 depicts the experimental approach in collecting the SHG adsorption isotherm data. In general, a known quantity of vapor, monitored using pressure sensors (WRG-S: IPV25MKA and Barocel: W60014811, Edwards High Vacuum Int.), was introduced into the evacuated airtight gas chamber, which contained liquid mercury. Vacuum was achieved by a rotary vane pump (Brook Compton, Inc.). The SHG intensity, $I_{\mathrm{SHG}}$, at $401 \mathrm{~nm}$ was collected after introducing the vapor into the chamber. The SHG intensity from the virgin mercury surface under vacuum, $I_{\mathrm{SHG}, \mathrm{Hg}}$, was used as a reference signal to normalize the total $I_{\mathrm{SHG}}$, which includes signal from the substrate and the substrate-adsorbate interaction. The normalized SHG, $E_{\mathrm{NormSHG}}=\sqrt{I_{\mathrm{SHG}} / I_{\mathrm{SHG}, \mathrm{Hg}}}$, was plotted against the relative pressure, $P / P^{0}$, where $P$ is the measured vapor pressure of the compound in equilibrium with the mercury surface and $P^{0}$ is the saturated vapor pressure of the compound. To collect adsorption isotherms, the entire vacuum line and the cell containing liquid mercury was evacuated to a pressure of $\sim 10^{-3}$ Torr for 20 minutes, while valve V3 was closed to the solvent. Next, V6 was closed and the SHG signal $\left(I_{\mathrm{SHG}, \mathrm{Hg}}\right)$ was measured from the virgin $\mathrm{Hg}_{(1)}$ surface. To introduce solvent vapor into the vacuum chamber V3 was opened while V2 was kept closed, followed by closing V3 and opening V2 to the entire vacuum line and the cell containing mercury. This allowed us to control the amount of vapor added into the chamber and was done in increments of a few Torr and the total pressure was read as $P_{\text {vapor }}$ using the pressure sensors. For each $P_{\text {vapor }}$ reading, a SHG signal $\left(I_{\mathrm{SHG}}\right)$ was collected.

\section{Chemicals}

Elemental mercury $\left(\mathrm{Hg}_{(1)}\right)$ was purchased from Sigma Aldrich $(\geq 99.99 \%$ trace metals basis). It was subject to a series of purification steps. First, $\mathrm{Hg}_{(1)}$ was transferred to a clean (washed with a diluted acid solution) and dry round bottom flask via

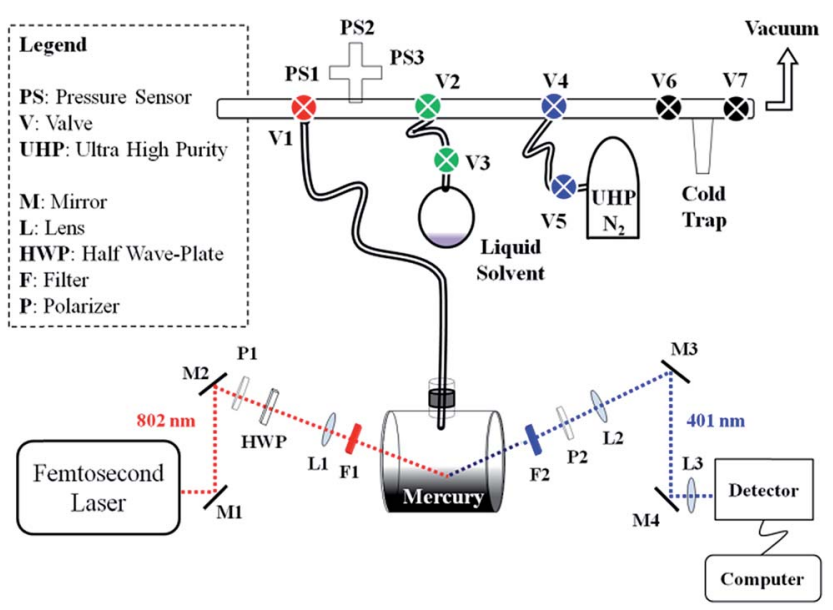

Fig. 1 Schematic of the experimental setup for monitoring the adsorption of vapor on mercury surface using second harmonic generation. multiple transferring steps. Each transferring step involved drawing liquid mercury from the interior of the bulk liquid from a vial using a plastic micropipette. Since $\mathrm{Hg}_{(1)}$ has a high surface tension, contaminants or particulate matter reside on the surface. Thus, a series of micropipette transfers ( 3 to 5 times) ensured that only the unexposed virgin $\mathrm{Hg}_{(1)}$ was taken and surface contaminants were left behind. After transferring, the mercury in the round bottom flask was subject to freeze-thaw purging with ultrahigh purity (UHP) nitrogen (99.999\% MEGS specialty gas) for at least 3 times. This batch of mercury served as our "stock" sample, which was stored in the dark. For the experiment, the stock sample was transferred to the spectroscopic cell (built in-house glass cell with quartz windows) using the micropipette transfer method described above. The cell was connected to the vacuum line (using Teflon tubes) and positioned under the laser light. Then the cell containing $\mathrm{Hg}_{(1)}$ was purged with UHP nitrogen for 3 to 5 times. The vapor to be adsorbed on pure mercury surface was produced in a vacuum line (Fig. 1). The liquid solvent to be studied was placed in a round bottom flask and purged (separately; not with $\mathrm{Hg}_{(1)}$ spectroscopic cell connected) with UHP $\mathrm{N}_{2}$ at least 3 times and then evacuated with V3 closed. The flask containing the solvent was kept in a water bath at constant temperature during the experiment.

\section{SHG setup}

The experimental configuration for SHG measurements consisted of a Nd: $\mathrm{YVO}_{4}$ solid state laser (Spectra-Physics, Millennia PRO 6sJ) pumped Ti:Sapphire Tsunami oscillator (SpectraPhysics, 3941-M1BB), which provided sub $100 \mathrm{fs}$ pulses at a repetition rate of $80 \mathrm{MHz}$. Fig. 1 depicts the experimental layout (not drawn to scale) for generating SHG signal from the mercury surface. In brief, the femtosecond laser pulse train was passed through a polarizer, a half-wave plate and then focused onto the surface at an angle of $70^{\circ}$, with respect to the normal. A red filter (F1) was used between the sample and the lens to block the stray light at twice the frequency of the laser light. A typical energy of $3.3 \mathrm{~nJ}$ per pulse at $802 \mathrm{~nm}$ was used to perform the experiments.

For the purpose of adsorption isotherm measurement, the incident beam was P-polarized; i.e., parallel to the normal of incidence plane. As shown in Fig. 1, the reflected light was guided through a blue filter (F2) to block out any residual 802 $\mathrm{nm}$ light to prevent further SHG from being generated from collection optics. The P-polarized SHG signal at $401 \mathrm{~nm}$ was selected using a polarizer (P2) and focused onto a monochromator (Spectral Products, DK480, $500 \mathrm{~mm}$ ) and thereafter, detected using a photomultiplier tube (PMT) (Spectral Products, R928P). The PMT output was then amplified (SR445A) and processed using a gated photon counter (SR400) (Stanford Research Systems) using the internal $10 \mathrm{MHz}$ clock as base. Using a home-built Labview program the SHG intensity was recorded as a function of time and then averaged. For null angle measurements, the detected signal was selected as P-polarized, while the incident beam polarization was varied using a half wave-plate (HWP). The average power of the incident beam at the sample for day to day was in the range of $250-270 \mathrm{~mW}$. 
During the collection of SHG data, power variation remained less than $1 \%$. The beam diameter was $3 \mathrm{~mm}$ (ca.) and focused onto the sample with a lens of $7.5 \mathrm{~cm}$ focal length.

\section{Data acquisition and analysis}

Data analysis was carried out using non-linear least-squares data fitting tools in Igor Pro 4.04. Adsorption isotherms were also fitted using the nonlinear regression analysis tools in Origin Pro 8, and cross-validated with the fitting parameters obtained using Igor Pro. Both Igor and Origin fitting tools used the Levenberg-Marquardt algorithm and produced comparable values for the fitting parameters and the associated uncertainties.

\section{Results}

For the aromatic compounds studied, it is found that the second harmonic signal from the liquid mercury surface increases upon the introduction of vapors (Fig. 2, left, red dots) into the chamber (Fig. 1). Because SHG does not originate from bulk isotropic media ${ }^{\mathbf{1 4 - 1 6 , 2 4}}$ such as the gaseous vapor phase, the change in the SHG signal corresponds to the adsorption and interaction of molecules with the mercury surface. Moreover, upon evacuating the cell (Fig. 2, right, green dots), the SHG intensity is found to decrease, due to desorption of molecules, back to the original signal level corresponding to neat liquid mercury surface indicating that these compounds undergo reversible physisorption and that no chemical reaction occurs at the $\mathrm{Hg}_{(1)}$ surface. It is clear from the kinetic data in Fig. 2, complete adsorption, when exposed to saturated vapor, as well as removal from the surface under vacuum take place in less than a minute for both benzene and toluene. The data presented in Fig. 2 correspond to the average of three independent kinetic runs. The multiple cycles of adsorption and desorption kinetic on the same $\mathrm{Hg}_{(1)}$ surface, which highlights the reversibility of benzene and toluene adsorption mercury surface, are shown in the Appendix (Fig. A.1†).

Further evidence that the surface of mercury remains unperturbed upon adsorption was discerned based on SHG null angle measurements. In this experiment, P-polarization SHG

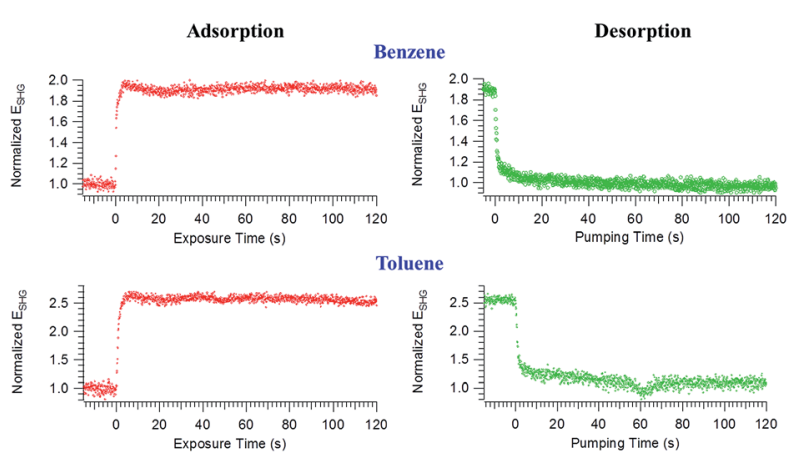

Fig. 2 Normalized SHG field for the kinetics of adsorption (left, red dots) and desorption (right, green dots) of benzene and toluene as a function of exposure time and pumping time, respectively. The experimental data correspond to the average of 3 independent trials. signal was detected and polarization of the incident beam was varied using a half-wave plate. It was found that the null angle, which is the polarization angle of the incident beam where the detected SHG signal at a fixed polarization (i.e., P-polarization) is a minimum, did not vary with molecular adsorption (Fig. 3). The null angle for the neat as well as the surface covered mercury under saturated vapor pressure, independent of the vapors studied, was obtained with S-polarized (i.e., horizontal with respect to the surface normal) incident beam. In addition, we did not observe any shift in the null angle at different surface coverages of either toluene or benzene. Since the null angle did not change with molecular adsorption, the possibility of change in the structural symmetry of mercury surface upon adsorption can be excluded. Furthermore, this indicates that the orientation of the adsorbed molecule and thereby, the relative phase does not influence the SHG signal as a function of molecular coverage.

Fig. 4 shows (red dots) normalized SHG, $E_{\text {NormsHG }}$, as a function of relative pressure, $P / P^{0}$, obtained upon addition of benzene (4a) and toluene (4b) vapors in the range of $289.5 \mathrm{~K}$ to 291.5 K. Several existing isotherm models (Langmuir (Lang), Brunauer-Emmett-Teller (BET), Volmer (Vol), FrumkinFowler-Guggenheim (FFG), and Hill-de Boer (HdB); see Appendix, eqn (A.1) to (A.5), $\dagger$ which describe the $\theta$ dependence on the relative pressure, were tested to describe the adsorption behavior. It is important to emphasize that because the null angle does not vary with surface coverage, $\theta$, the SHG field can be considered as directly proportional to the number of molecules present at the surface. ${ }^{\mathbf{1 9 , 2 1 , 2 2}}$ In general, formation of new products (i.e., appearance of new electronic states) or molecular

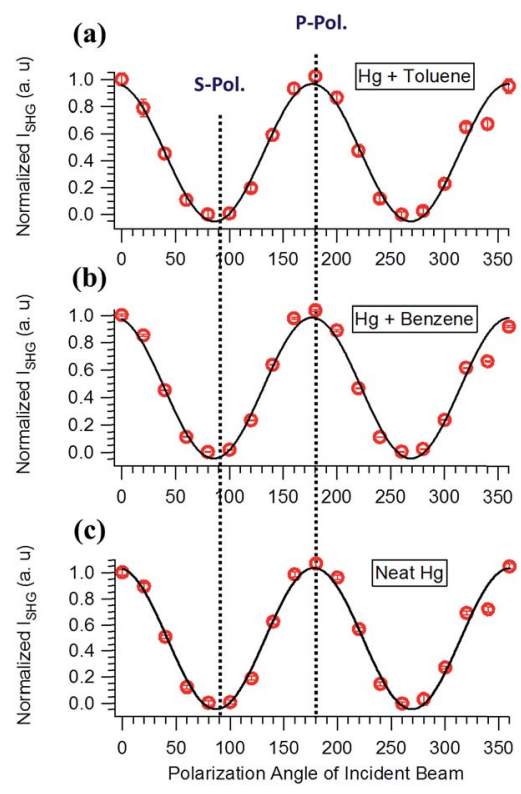

Fig. 3 Normalized SHG intensity as a function of polarization angle of the incident beam from different surfaces of mercury exposed to: (a) 20.1 Torr of toluene, (b) 63.1 Torr of benzene, and (c) vacuum ( $10^{-3}$ Torr). The solid black curve is an empirical fit to guide the eye. To normalize, the SHG intensity detected was divided by the maximum intensity observed when the incident beam was P-polarized. 
(a)

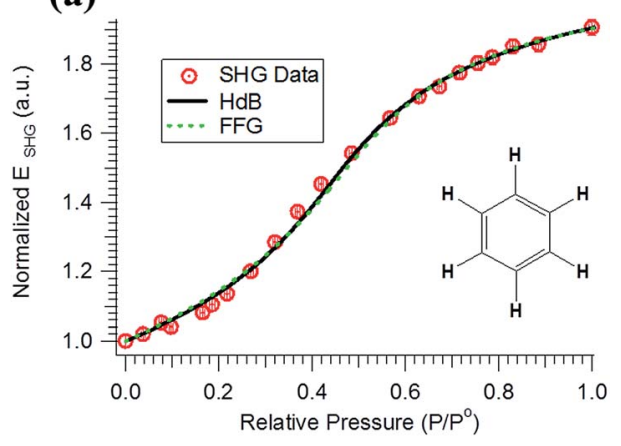

(b)

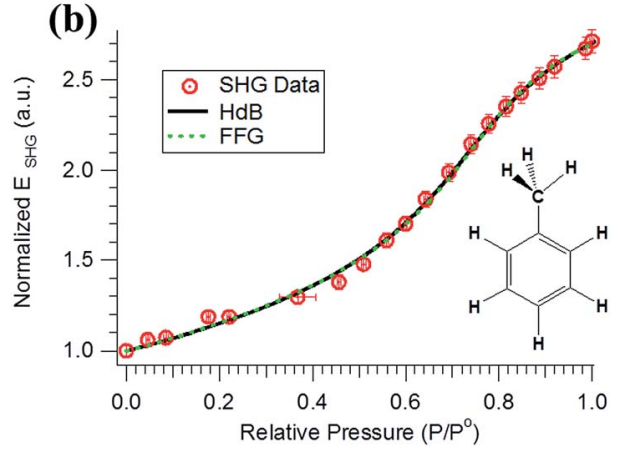

Fig. 4 SHG adsorption isotherms of (a) benzene and (b) toluene. The experimental data (shown as red circles) are the normalized SHG field, the solid black line and dashed green line represent Hill-de Boer and Frumkin-Fowler-Guggenheim fits to the experimental data, respectively.

orientation change can lead to a shift in the relative phase. As mentioned earlier, the laser and the SHG frequency is offresonant with the species investigated and there is no indication of product formation for this adsorption process (Fig. 2). Due to this fact and the evidence that there is no orientation change (Fig. 3), it is reasonable to assume that the relative phase is not changing with molecular coverage. Thus, the plots in Fig. 4 can be considered as adsorption isotherms of the aromatic vapors on $\mathrm{Hg}_{(1)}$ surface and the existing isotherm models can be applied. The isotherm equations for all of the models considered and the experimental data for all the trials along with the fit results are shown in the Appendix (eqn (A.1) to (A.5) and Fig. A.2†). A representative set of data is shown in Fig. 4. When applying the $\mathrm{Vol}, \mathrm{FG}$, and $\mathrm{HdB}$ isotherm equations, it was necessary to numerically fit $E_{\mathrm{SHG}} v s . P / P^{0}$ data because it is not possible to obtain an analytical solution of $\theta$ for these models.

\section{Discussions}

Based on our data analysis, the following conclusions are reached: (a) first, Langmuir, Volmer, and BET models did not fit the experimental results for the compounds investigated (see Appendix, Fig. A.2†). These isotherm models ${ }^{40,41}$ do not take into account of the adsorbate-adsorbate lateral interaction. Both Langmuir and BET models assume localized adsorption, whereas, the Volmer equation considers adsorbed molecules as mobile. BET incorporates multilayer adsorption; that is, while localized, more than one molecule can adsorb with different affinities, one on top of the other; ${ }^{40}$ (b) second, the adsorption of benzene and toluene vapors onto liquid mercury surface can be described using the Hill-de Boer isotherm model, which takes into account of lateral interaction and considers the adsorbates to be mobile. The Frumkin-Fowler-Guggenheim model, which is a modification of the Langmuir isotherm model incorporating lateral interaction, fits the data as well. These findings indicate that lateral interaction between adsorbed species is substantial for the adsorption of benzene and toluene.

The dashed green line and the solid black line in Fig. 4 are the FFG (eqn (4)) and HdB (eqn (5)) fits, respectively, of the experimental data.

$$
\begin{gathered}
K_{\mathrm{FFG}} P=\frac{\theta}{(1-\theta)} \mathrm{e}^{-w \theta} \\
K_{\mathrm{HdB}} P=\frac{\theta}{1-\theta} \exp \left[\frac{\theta}{1-\theta}-\frac{2 \alpha \theta}{k_{\mathrm{B}} T \beta}\right]
\end{gathered}
$$

The averages of the fit parameter values from 3 different independent trials (see Appendix, Fig. A. $2 \dagger$ ) for these compounds are provided in Table 1. The FFG isotherm (eqn (4)) is based on the same assumptions as Langmuir model; however, lateral interactions between the neighboring adsorbates are taken into account. The positive value of the interaction parameter, $w$, for both benzene and toluene indicates that the lateral interactions are attractive in nature, with benzene $(w=2.4 \pm 0.2)$ experiencing relatively weaker lateral interaction than toluene $(w=3.3 \pm 0.3)$. A similar conclusion is reached when the Hill-de Boer equation for mobile adsorption is used.

The HdB isotherm (eqn (5)) is the two-dimensional analog of the van der Waals equation ${ }^{40}$ and is derived based on the Gibbs adsorption theorem that relates the gas phase equilibrium pressure to the spreading pressure of the adsorbed film. It is equally applicable in describing adsorption on a homotattic surface above and below the two-dimensional critical temperature (562 K and $592 \mathrm{~K}$ for benzene and toluene, respectively). ${ }^{42}$ In this model adsorbates are mobile and exhibit lateral interaction with neighboring molecules. The parameters $\alpha$ and $\beta$ are the twodimensional analogs of van der Waals constants $a$ and $b$, respectively. The quantity $2 \alpha / \beta$, known as the interaction energy, reflects the lateral interaction between adsorbed molecules. For both benzene and toluene this ratio is positive, which denotes that these species exhibit attractive adsorbate-adsorbate interaction.

Based on the HdB parameters it is possible to gain further insight into the surface-adsorbate interaction. It has been shown $^{44,45}$ that if the lateral interaction by the substrate, i.e., surface mercury atoms in our case, and the particular orientation of the adsorbate toward the surface are neglected, then the two-dimensional and three-dimensional constants are related as follows: $2 \alpha / \beta=a / b$. Table 1 gives the $a / b$ values, where $a$ is a measure of the attraction between the molecule and $b$ is the excluded volume. Comparison of $a / b$ value to the corresponding $2 \alpha / \beta$ value is expressed as the $\frac{2 \alpha / \beta}{a / b}$ ratio. This ratio shows that 
Table 1 Hill-de Boer and Frumkin-Fowler-Guggenheim adsorption isotherm fit parameters and van der Waals gas parameters ${ }^{43}$ of benzene and toluene

\begin{tabular}{|c|c|c|c|c|c|c|c|c|c|c|c|}
\hline Compounds & \multicolumn{3}{|c|}{ HdB fit parameters } & \multicolumn{3}{|c|}{ FFG fit parameters } & \multicolumn{2}{|c|}{ van der Waals parameters } & $\frac{2 \alpha / \beta}{a / b}$ & $\begin{array}{l}\text { Vapor } \\
\text { pressure } \\
P^{0} \text { (Torr) }\end{array}$ & $\begin{array}{l}\text { Experimental } \\
\text { temperature } \\
(\mathrm{K})\end{array}$ \\
\hline Benzene & $1.7 \pm 0.2$ & $5.8 \pm 0.3$ & $2.6 \pm 0.2$ & $1.2 \pm 0.1$ & $9.0 \pm 0.4$ & $2.4 \pm 0.2$ & 18.24 & 0.1154 & $0.68 \pm 0.05$ & 63.3 & $289.7 \pm 0.5$ \\
\hline Toluene & $2.9 \pm 0.5$ & $8.3 \pm 1.1$ & $3.3 \pm 0.2$ & $2.0 \pm 0.3$ & $11 \pm 2$ & $3.3 \pm 0.3$ & 24.38 & 0.1463 & $0.81 \pm 0.06$ & 20.1 & $290.7 \pm 0.5$ \\
\hline
\end{tabular}

there is a decrease of $32( \pm 5) \%$ for benzene and $19( \pm 6) \%$ for toluene in the interaction energy due to the influence of the surface. The greater degree of decrease for benzene is due to the possibility of its pi-electrons experiencing a complete interaction with the mercury surface. In the case of toluene, the methyl substituent not only reduces the pi-electron interaction with the surface but also increases the probability of intermolecular lateral interaction, which manifests into a larger value of $2 \alpha / \beta$. Our result is in agreement with a similar trend previously observed with respect to interactions of pi-electrons at the vapor/solid interface. ${ }^{45}$ For instance, a larger decrease in the $\frac{2 \alpha / \beta}{a / b}$ ratio for unsaturated hydrocarbon (i.e., compounds containing double bonds) relative to saturated hydrocarbons has been attributed to the interaction of double bond with the surface of graphitized carbon. ${ }^{45}$

By fitting the experimental data using the $\mathrm{HdB}$ and FFG isotherm model, it can be concluded that lateral interaction is involved in the adsorption of benzene and toluene. Mobile adsorption is likely but it is not possible to discriminate mobile and localized monolayers rigorously based on the fits alone. ${ }^{40}$ However, the $\mathrm{HdB}$ isotherm equation provides a slightly lesser degree of uncertainty in the fit parameters compared to the FFG model for both benzene and toluene. Mobility has been hypothesized for benzene in earlier studies. ${ }^{34,36,46,47}$ Based on entropy of adsorption calculations, benzene at $298 \mathrm{~K}$ was considered to exhibit 2-dimensional rotation in the plane of the surface only. Toluene has been interpreted as not freely mobile or as experiencing lesser degree of mobility compared to benzene. ${ }^{34,36,46}$ The results obtained using SHG techniques are thus consistent with the earlier studies based on macroscopic surface tension measurements for these compounds. By interpreting the benzene and toluene SHG adsorption data we are further able to quantify the extent of lateral interactions that toluene $(w=3.3 \pm 0.3)$ and benzene $(w=2.4 \pm 0.2)$ exhibit when adsorbed at the $\mathrm{Hg}_{(1)}$ surface. Thus, the results reported not only advance the applicability of the SHG spectroscopy but provide a direct approach to probe vapor/liquid interface to obtain a quantitative understanding of the adsorption mechanism.

In summary, based on second harmonic generation experiments we demonstrated that both benzene and toluene undergo reversible physisorption, with no chemical reaction at the surface of liquid mercury in the range of $10^{-3}$ Torr up to saturated vapor pressure of the compound, at room temperature. It is found that the surface interaction of these aromatic
VOCs can be described by the HdB and FFG models, which indicates that lateral interactions is dominant for the adsorption of these species onto the liquid mercury surface. Both benzene and toluene exhibit attractive lateral interactions.

\section{Conclusions}

The work presented extends the applicability of SHG to investigate complex vapor to liquid adsorption processes that involve lateral and mobile adsorbate-surface interactions. This capability of monitoring adsorption mechanisms and adsorption kinetics under ambient condition will be particularly useful in characterizing the adsorption of atmospheric pollutants, such as VOCs, to the air/aqueous interface. Molecular level understanding of VOCs adsorption onto air/aqueous, vapor/aerosol, and gas/particle interfaces is necessary to better understand the atmospheric fate and transport and subsequent reactivity of these compounds..$^{7-9}$ For instance, the rate and mechanism by which VOCs adsorb and react onto water droplets or surface of aerosol particles will dictate how far and fast these pollutants can be transported in the atmosphere. Liquid mercury, with its atomically smooth and homogeneous surface, resembles the air/water interface in many respects and thus serves as an ideal platform to understand these adsorption mechanisms.

Previously, surface selective spectroscopy has been applied to probe environmental surfaces ${ }^{23,25}$ and this work further advances the potential of this technique to elucidate fundamental knowledge of the adsorption of atmospherically relevant vapors on an ideally homogeneous surface.

\section{Acknowledgements}

M. Subir gratefully acknowledges Dr Yi Rao for fruitful discussions and for his kind support. We also thank Environment Canada, Natural Science and Engineering Research Council of Canada and Ball State University for their financial support and resources.

\section{Notes and references}

1 K. T. Valsaraj, Pure Appl. Chem., 2009, 81, 1889-1901.

2 K.-U. Goss, Crit. Rev. Environ. Sci. Technol., 2004, 34, 339-389.

3 K.-U. Goss and S. J. Eisenreich, Environ. Sci. Technol., 1996, 30, 2135-2142. 
4 R. J. Blaszczak, Choosing an Adsorption System for VOC: Carbon, Zeolite, or Polymers?, United State Environmental Protection Agency, EPA 456/F-99-004, 1999.

5 N. A. Eltouny and P. A. Ariya, Ind. Eng. Chem. Res., 2012, 51, 12787-12795.

6 G. R. Parmar and N. N. Rao, Crit. Rev. Environ. Sci. Technol., 2008, 39, 41-78.

7 V. H. Grassian, Int. Rev. Phys. Chem., 2001, 20, 467-548.

8 D. J. Donaldson and K. T. Valsaraj, Environ. Sci. Technol., 2010, 44, 865-873.

9 M. T. C. Martins-Costa, J. M. Anglada, J. S. Francisco and M. F. Ruiz-Lopez, J. Am. Chem. Soc., 2012, 134, 11821-11827.

10 R. Lohmann and G. Lammel, Environ. Sci. Technol., 2004, 38, 3793-3803.

11 E. C. Griffith, T. R. C. Guizado, A. S. Pimentel, G. S. Tyndall and V. Vaida, J. Phys. Chem. C, 2013, 117, 22341-22350.

12 J.-R. Li, Y. Ma, M. C. McCarthy, J. Sculley, J. Yu, H.-K. Jeong, P. B. Balbuena and H.-C. Zhou, Coord. Chem. Rev., 2011, 255, 1791-1823.

13 M. T. Luebbers, T. Wu, L. Shen and R. I. Masel, Langmuir, 2010, 26, 11319-11329.

14 C.-Y. Huang, M. Song, Z.-Y. Gu, H.-F. Wang and X.-P. Yan, Environ. Sci. Technol., 2011, 45, 4490-4496.

15 A. J. Fletcher, E. J. Cussen, D. Bradshaw, M. J. Rosseinsky and K. M. Thomas, J. Am. Chem. Soc., 2004, 126, 9750-9759.

16 K. B. Eisenthal, Chem. Rev., 2006, 106, 1462-1477.

17 K. B. Eisenthal, Chem. Rev., 1996, 96, 1343-1360.

18 A. B. Voges, H. A. Al-Abadleh and F. M. Geiger, in Environmental Catalysis, ed. V. H. Grassian, Taylor \& Francis, Boca Raton, 2005.

19 Y. R. Shen, The Principles of Nonlinear Optics, John Wiley \& Sons, Inc, Hoboken, 2003.

20 G. L. Richmond, H. M. Rojhantalab, J. M. Robinson and V. L. Shannon, J. Opt. Soc. Am. B, 1987, 4, 228-236.

21 G. L. Richmond, J. M. Robinson and V. L. Shannon, Prog. Surf. Sci., 1988, 28, 1-70.

22 J. F. McGilp, Surf. Rev. Lett., 1999, 06, 529-558.

23 A. M. Jubb, W. Hua and H. C. Allen, Annu. Rev. Phys. Chem., 2012, 63, 107-130.

24 G. Gonella and H.-L. Dai, Langmuir, 2013, 30, 2588-2599.

25 F. M. Geiger, Annu. Rev. Phys. Chem., 2009, 60, 61-83.
26 R. M. Corn and D. A. Higgins, Chem. Rev., 1994, 94, 107-125. 27 W.-k. Zhang, H.-f. Wang and D.-s. Zheng, Phys. Chem. Chem. Phys., 2006, 8, 4041-4052.

28 J. Liu, M. Subir, K. Nguyen and K. B. Eisenthal, J. Phys. Chem. $B, 2008,112,15263-15266$.

29 M. Subir, J. Liu and K. B. Eisenthal, J. Phys. Chem. C, 2008, 112, 15809-15812.

30 M. Wilkinson, Chem. Rev., 1972, 72, 575-625.

31 L. Tamam, H. Kraack, E. Sloutskin, B. M. Ocko, P. S. Pershan, E. Ofer and M. Deutsch, J. Phys. Chem. C, 2007, 111, 2573-2579.

32 L. Tamam, H. Kraack, E. Sloutskin, B. M. Ocko, P. S. Pershan, E. Ofer and M. Deutsch, J. Phys. Chem. C, 2007, 111, 2580-2587.

33 C. Sanford and S. Ross, J. Phys. Chem., 1954, 58, 288.

34 C. Kemball, Proc. R. Soc. London, Ser. A, 1946, 187, 73-87.

35 C. Kemball, Proc. R. Soc. London, Ser. A, 1950, 201, 377-391.

36 C. Kemball and E. K. Rideal, Proc. R. Soc. London, Ser. A, 1946, 187, 53-73.

37 P. Galletto, S. Loridant, R. Antoine, P. F. Brevet and H. H. Girault, J. Electroanal. Chem., 2000, 487, 16-24.

38 F. Eisert, A. P. Elg and A. Rosén, Appl. Phys. A, 1995, 60, 209215.

39 R. W. Boyd, Nonlinear Optics, Academic Press, Rochester, 2003.

40 J. Lyklema, Fundamentals of Interface and Colloid Science, Academic Press, London, 1995.

41 D. Myers, Surface, Interfaces and Colloids, Wiley-VCH, New York, 1999.

42 W. D. Machin and S. Ross, Proc. R. Soc. London, Ser. A, 1962, 265, 455-462.

43 R. C. Weast, Handbook of Chemistry and Physics, Chemical Rubber Co., Cleveland, 53rd edn, 1972.

44 J. H. Boer, The dynamical character of adsorption, Clarendon P., 1968.

45 S. E. Hoory and J. M. Prausnitz, Trans. Faraday Soc., 1967, 63, 455-460.

46 T. L. Hill and C. Kemball, J. Am. Chem. Soc., 1952, 74, 39463948.

47 B. A. Pethica and M. L. Glasser, Langmuir, 2004, 21, 944-949. 